\title{
Proposta de uma Abordagem Iterativa Analítica para o Oscilador Harmônico Simples
}

\author{
Proposal of an Analytical Iterative Approach for the Simple Harmonic Oscillator \\ Breno Rodrigues Segatto*10 \\ ${ }^{1}$ Universidade Federal do Espírito Santo, Departamento de Ciências Naturais, Campus São Mateus, ES, Brasil.
}

Recebido em 12 de Dezembro de 2018. Revisado em 24 de Agosto de 2019. Aceito em 09 de Setembro de 2019.

\begin{abstract}
Neste trabalho propusemos uma abordagem iterativa analítica em que a evolução temporal foi substituída pelo número de iterações. E para o caso de um sistema massa-mola obtivemos sua velocidade e sua posição em função do tempo como somas parciais de séries que convergiram para as funções trigonométricas que são as soluções do oscilador harmônico simples.
\end{abstract}

Palavras-chave: Oscilador Harmônico Simples, Método Analítico, Mecânica.

In this work we propose an analytical iterative approach in which the temporal evolution was replaced by the number of iterations. And for the case of a mass-spring system we obtained its velocity and its position as a function of time as partial sums of series that converged to the trigonometric functions that are the solutions of the simple harmonic oscillator.

Keywords: Simple Harmonic Oscillator, Analytical Method, Mechanics.

\section{Introdução}

Muitas vezes o professor em sala de aula tenta convencer e demonstrar aos seus estudantes que a ciência, neste caso em questão a física, é algo em constante evolução, e que as ciências naturais buscam a compreensão da natureza. Essa compreensão baseia-se especificamente na modelagem dos fenômenos físicos. Muitos recursos vem sendo desenvolvidos com o intui-to de avançar no processo de ensino-aprendizagem [1-4], principalmente recursos computacionais 5 9 e experimentais $[10 \sqrt{12}$. Propõe-se então neste trabalho, um modelo analítico alternativo para obtenção das funções horárias do oscilador harmônico simples (ohs) 22] como recurso didático, levantando a questão da problematização de uma proposta analítica alternativa às usuais. É importante ressaltar que o intuito não é desenvolver um método melhor nem de mais fácil resolução, e sim um método alternativo, no qual possamos obter as funções horárias para o ohs sem a necessidade de resolvermos diretamente as equações diferencias ou utilizar métodos numéricos ou modelagens computacionais. A escolha do ohs foi pelo fato de além do mesmo ser utilizado em uma vasta gama de sistemas físicos [13 21], é um problema simples e sua solução é dada em termos de funções ele-mentares (equações (3) e (4)). E devido a isto, é frequentemente abordado em textos de física básica 22,23]. Uma das maneiras de resolver o ohs é partindo da Lei de Hooke [23 para obter suas funções horárias $x(t)$ e $v(t)$. Assim

*Endereço de correspondência: breno.segatto@ufes.br

$$
\begin{aligned}
& m \frac{d^{2} x}{d t^{2}}+k x=0 \\
& \frac{d^{2} x}{d t^{2}}+w^{2} x=0
\end{aligned}
$$

a equação (2) é uma equação diferenciável homogênea de segunda ordem, onde $w=\sqrt{\frac{k}{m}}$ é a frequência natural do sistema massa-mola e sua solução é do tipo

$$
x(t)=A \operatorname{sen}(w t)+B \cos (w t)
$$

com

$$
v(t)=\frac{d x(t)}{d t}=A w \cos (w t)-B w \operatorname{sen}(w t),
$$

onde A e B podem ser determinados pelas condições iniciais $x(0)=x_{0}$ e $v(0)=v_{0}$, resultando em

$$
x(t)=\frac{v_{0}}{w} \operatorname{sen}(w t)+x_{0} \cos (w t)
$$

$\mathrm{e}$

$$
v(t)=v_{0} \cos (w t)-x_{0} w \operatorname{sen}(w t) .
$$

As equações (5) e (6) são as funções horárias para o ohs. Na seção a seguir iremos descrever uma abordagem iterativa analítica para obtermos estas equações como um recurso alternativo. 


\section{Abordagem Iterativa para o Oscilador Harmônico Simples}

Vamos considerar aqui um sistema massa-mola clássico, e consideraremos o movimento relativo da massa reduzida $m$ em um sistema de dois corpos. Em um tempo inicial $t_{0}$ o sistema tem posição inicial $x_{0}$ e velocidade inicial $v_{0}$, o sistema se propaga livremente em uma trajetória retilínea até a sua primeira iteração subsequente em

$$
t_{1}=t_{0}+\Delta t_{0}
$$

e

$$
x_{1}=x_{0}+\Delta x_{0}=x_{0}+v_{0} \Delta t_{0}
$$

onde muda sua velocidade para

$$
v_{1}=v_{0}+\Delta v_{0}
$$

O sistema segue, novamente, uma trajetória retilínea até sua segunda iteração em

$$
t_{2}=t_{1}+\Delta t_{1}
$$

O qual muda sua velocidade para

$$
v_{2}=v_{1}+\Delta v_{1} \text {. }
$$

Assim na enésima iteração

$$
\begin{gathered}
t_{n}=t_{0}+\sum_{j=0}^{n-1} \Delta t_{j} \\
v_{n}=v_{n-1}+\Delta v_{n-1}=v_{0}+\sum_{j=0}^{n-1} \Delta v_{j}
\end{gathered}
$$

$\mathrm{e}$

$$
x_{n}=x_{n-1}+\Delta x_{n-1}=x_{0}+\sum_{j=0}^{n-1} v_{j} \Delta t_{j} .
$$

$\mathrm{O}$ vetor posição $\vec{x}_{n}=x_{n} \hat{i}$, muda continuamente, descrevendo uma trajetória poligonal. Nesta abordagem a velocidade (momento linear se quisermos dar um tratamento semi-clássico 24]) muda discretamente. As variáveis contínuas tempo e posição entram na descrição do movimento como se fossem parâmetros discretos somente porque esses eventos de iteração são nossos pontos de referência para a contagem do tempo. As equações 13 e (14) são relações genéricas válidas para qualquer sistema não relativístico discreto. Para o ohs considerado aqui é natural esperarmos que

$$
\Delta p_{n}=-k x_{n-1} \Delta t_{n-1} .
$$

Uma vez que, $\Delta p_{n}=m \Delta v_{n-1}$ e $w=\sqrt{\frac{k}{m}}$ temos

$$
\Delta v_{n}=-w^{2} x_{n-1} \Delta t_{n-1}
$$

o qual define nosso ohs discreto. Dentre infinitas possibilidades nós iremos escolher a mais simples matematicamente

$$
\Delta t_{n} \equiv \alpha
$$

$$
\Delta v_{n} \equiv-\alpha w^{2} x_{n-1},
$$

onde $\alpha$ é uma constante positiva. As equações 13 e 14 tornam-se então

$$
v_{n}=v_{0}-\alpha w^{2} \sum_{j=0}^{n-1} x_{j}
$$

$$
x_{n}=x_{0}+\alpha \sum_{j=0}^{n-1} v_{j} .
$$

A combinação recursiva das equações 19 e 20 leva a

$$
x_{n}=\sum_{s=0}^{\left[\frac{n}{2}\right]}(\alpha w)^{2 s} x_{n}^{(s)}
$$

e

$$
v_{n}=\sum_{s=0}^{\left[\frac{n}{2}\right]}(\alpha w)^{2 s} v_{n}^{(s)},
$$

onde $x_{n}^{(s)}$ e $v_{n}^{(s)}$ são funções polinomiais de $x_{0}$ e $v_{0},\left[\frac{n}{2}\right]$ é o maior inteiro em $n / 2$, com

$$
\begin{gathered}
x_{n}^{(0)}=x_{0}+v_{0} \sum_{j_{1}=0}^{n-1} \alpha=x_{0}+v_{0} \alpha n, \\
v_{n}^{(0)}=v_{0}-w^{2} x_{0} \alpha n, \\
x_{n}^{(s)}=-\sum_{j_{1}=0}^{n-1} \sum_{j_{2}=0}^{j_{1}-1} x_{j_{2}}^{(s-1)}
\end{gathered}
$$

$$
v_{n}^{(s)}=-\sum_{j_{1}=0}^{n-1} \sum_{j_{2}=0}^{j_{1}-1} v_{j_{2}}^{(s-1)},
$$

$x_{n}^{(0)}$ seria a posição no instante $t_{n}$ se não houvesse iteração entre $t_{0}$ e $t_{n}$, enquanto $v_{n}^{(0)}$ seria a velocidade no instante $t_{n}$ se todas $n$ iterações forem iguais a primeira; $x_{n}^{(1)}$ seria a posição final se houvesse apenas uma iteração neste intervalo de tempo, e assim por diante. Sucessivas re-iterações nas equações 25 e 26 tornam se

$$
\begin{aligned}
x_{n}^{(s)} & =(-1)^{s} \sum_{j_{1}=0}^{n-1} \sum_{j_{2}=0}^{j_{1}-1} \cdots \sum_{j_{2 s}=0}^{j_{2 s-1}-1} x_{j_{2 s}}^{(0)} \\
& =(-1)^{s} \sum_{j_{1}=0}^{n-1} \sum_{j_{2}=0}^{j_{1}-1} \cdots \sum_{j_{2 s}=0}^{j_{2 s-1}-1}\left(x_{0}+v_{0} \alpha j_{2 s}\right)(27)
\end{aligned}
$$




$$
\begin{aligned}
& v_{n}^{(s)}=(-1)^{s} \sum_{j_{1}=0}^{n-1} \sum_{j_{2}=0}^{j_{1}-1} \cdots \sum_{j_{2 s}=0}^{j_{2 s-1}-1} v_{j_{2 s}}^{(0)} \\
& =(-1)^{s} \sum_{j_{1}=0}^{n-1} \sum_{j_{2}=0}^{j_{1}-1} \cdots \sum_{j_{2 s}=0}^{j_{2 s-1}-1}\left(v_{0}-\alpha w^{2} x_{0} j_{2 s}\right) .
\end{aligned}
$$

Usando agora $j_{2 s}=\left(\begin{array}{c}j_{2 s} \\ 1\end{array}\right)$ e a identidade

$$
\sum_{j=0}^{n-1}\left(\begin{array}{l}
j \\
k
\end{array}\right)=\left(\begin{array}{c}
n \\
k+1
\end{array}\right) \text { para } n>k
$$

(vide Apêndice A), temos

$$
x_{n}^{(s)}=(-1)^{s}\left(x_{0}\left(\begin{array}{c}
n \\
2 s
\end{array}\right)+\alpha v_{0}\left(\begin{array}{c}
n \\
2 s+1
\end{array}\right)\right)
$$

$$
v_{n}^{(s)}=(-1)^{s}\left(v_{0}\left(\begin{array}{c}
n \\
2 s
\end{array}\right)-\alpha w^{2} x_{0}\left(\begin{array}{c}
n \\
2 s+1
\end{array}\right)\right),
$$

substituindo 30 e 31 em 21 e 22 , obtemos

$$
\begin{aligned}
x_{n} & =\sum_{s=0}^{\left[\frac{n}{2}\right]}(-1)^{s}\left[(\alpha w)^{2 s} x_{0}\left(\begin{array}{c}
n \\
2 s
\end{array}\right)+(\alpha w)^{2 s+1}\right. \\
& \left.\times \frac{v_{0}}{w}\left(\begin{array}{c}
n \\
2 s+1
\end{array}\right)\right]
\end{aligned}
$$

$\mathrm{e}$

$$
\begin{aligned}
v_{n} & =\sum_{s=0}^{\left[\frac{n}{2}\right]}(-1)^{s}\left[(\alpha w)^{2 s} v_{0}\left(\begin{array}{c}
n \\
2 s
\end{array}\right)-(\alpha w)^{2 s+1}\right. \\
& \left.\times w x_{0}\left(\begin{array}{c}
n \\
2 s+1
\end{array}\right)\right] .
\end{aligned}
$$

Podemos reescrever as equações 32 e 33 da seguinte forma

$$
x_{n}=x_{0} f_{n}(\alpha w)+\frac{v_{0}}{w} g_{n}(\alpha w)
$$

$$
v_{n}=v_{0} f_{n}(\alpha w)-w x_{0} g_{n}(\alpha w)
$$

onde

$$
f_{n}(\alpha w) \equiv \sum_{s=0}^{\left[\frac{n}{2}\right]}(-1)^{s}(\alpha w)^{2 s}\left(\begin{array}{c}
n \\
2 s
\end{array}\right)
$$

$$
g_{n}(\alpha w) \equiv \sum_{s=0}^{\left[\frac{n}{2}\right]}(-1)^{s}(\alpha w)^{2 s+1}\left(\begin{array}{c}
n \\
2 s+1
\end{array}\right) .
$$

Para $n>1$, entretanto, a seguinte aproximação é válida

$$
\left(\begin{array}{l}
n \\
k
\end{array}\right) \approx \frac{n^{k}}{k !}
$$

(vide Apêndice B) e então

$$
\begin{aligned}
\sum_{s=0}^{\left[\frac{n}{2}\right]}(-1)^{s}(\alpha w)^{2 s}\left(\begin{array}{c}
n \\
2 s
\end{array}\right) & \approx \sum_{s=0}^{\left[\frac{n}{2}\right]}(-1)^{s}(\alpha w)^{2 s} \frac{n^{2 s}}{(2 s) !} \\
f_{n>>1}(\alpha w) & \approx \cos (\alpha w n)
\end{aligned}
$$

e

$$
\begin{aligned}
& \sum_{s=0}^{\left[\frac{n}{2}\right]}(-1)^{s}(\alpha w)^{2 s+1}\left(\begin{array}{c}
n \\
2 s+1
\end{array}\right) \approx \sum_{s=0}^{\left[\frac{n}{2}\right]}(-1)^{s}(\alpha w)^{2 s+1} \\
& \times \frac{n^{2 s+1}}{(2 s+1) !} \\
& g_{n>>1}(\alpha w) \approx \operatorname{sen}(\alpha w n) .
\end{aligned}
$$

Os últimos somatórios das equações 39 e e 40 são somas parciais das séries representando suas respectivas funções trigonométricas 25].

Os gráficos destas somas parciais estão mostrados nas Fig. 1 e Fig. 2 para $n=25$, Fig. 3 e Fig. 4 para $n=50$ e Fig. 5 e Fig. 6 para $n=100$. Observamos nestas figuras que $f_{n}(\alpha w)$ e $g_{n}(\alpha w)$ convergem inicialmente para suas respectivas funções trigonométricas $\cos (w t)$ e $\operatorname{sen}(w t)$ somente para valores de $\alpha w<<1$, uma vez que, o módulo das amplitudes de oscilação de $f_{n}(\alpha w)$ e $g_{n}(\alpha w)$ são muito maiores que um para os demais valores de $\alpha w$.

Entretanto, analiticamente os somatórios das equações (39) e 40 podem ser vistos como limites assintóticos

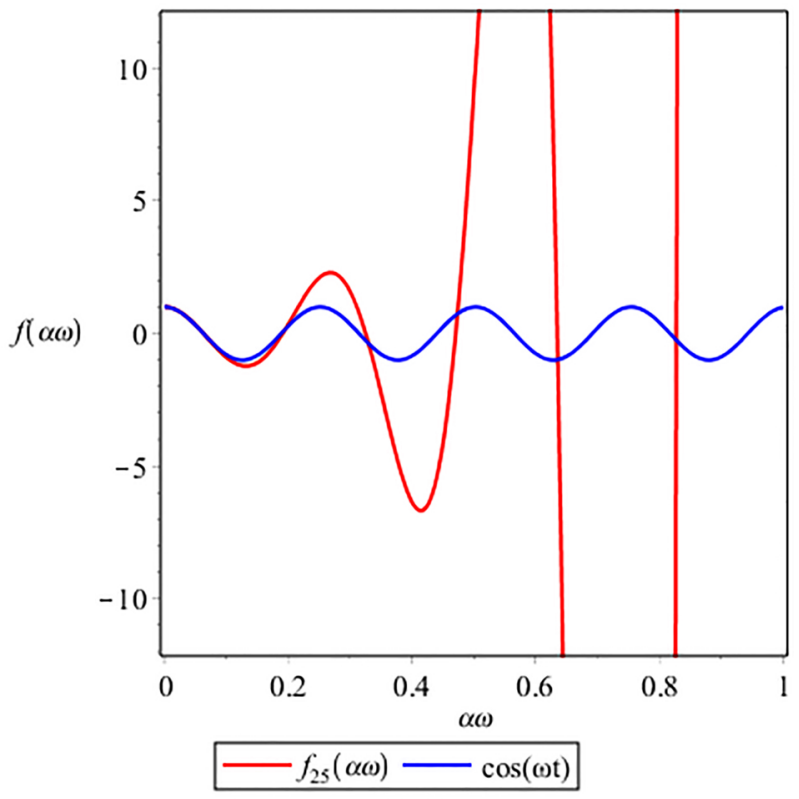

Figura 1: Amplitude $f(\alpha w)$ (que pode ser interpretada como $\frac{x_{25}}{x_{0}}$ quando $\left.v_{0}=0\right)$ e sua respectiva função trigonométrica. 


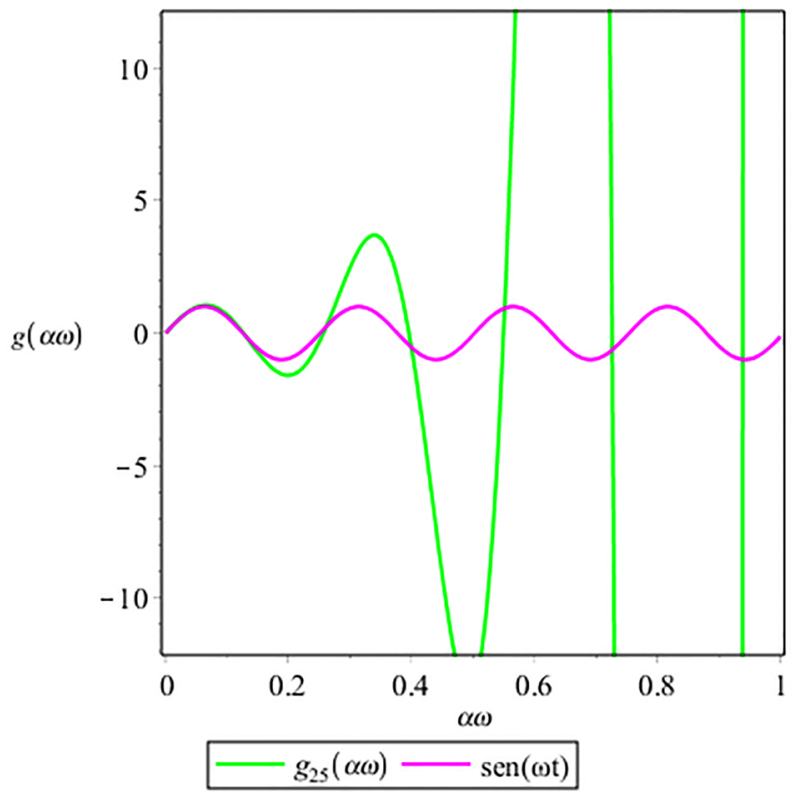

Figura 2: Amplitude $g(\alpha w)\left(\frac{-v_{25}}{w x_{0}}\right.$ para $\left.v_{0}=0\right)$ e sua respectiva função trigonométrica.

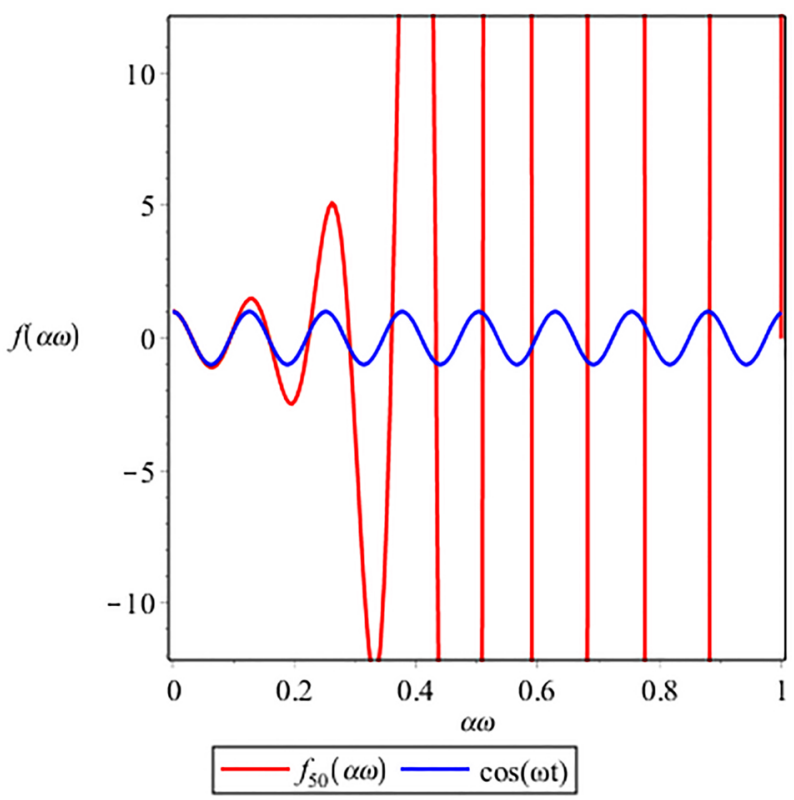

Figura 3: Amplitude $f(\alpha w)$ para $n=50$ e sua respectiva função trigonométrica. Vemos claramente que as amplitudes dimi-nuem para $\alpha w$ maiores quando comparados com $n=25$.

quando $n \rightarrow \infty$ em suas respectivas séries finitas de combinatoriais. E as equações 32 e 33 tornam-se soluções gerais do ohs.

$$
x_{n}=\frac{v_{0}}{w} \operatorname{sen}(w \alpha n)+x_{0} \cos (w \alpha n)
$$

e

$$
v_{n}=v_{0} \cos (w \alpha n)-x_{0} w \operatorname{sen}(w \alpha n) .
$$

As equações 12 e (17) definem a evolução temporal na abordagem iterativa aqui proposta, entretanto,

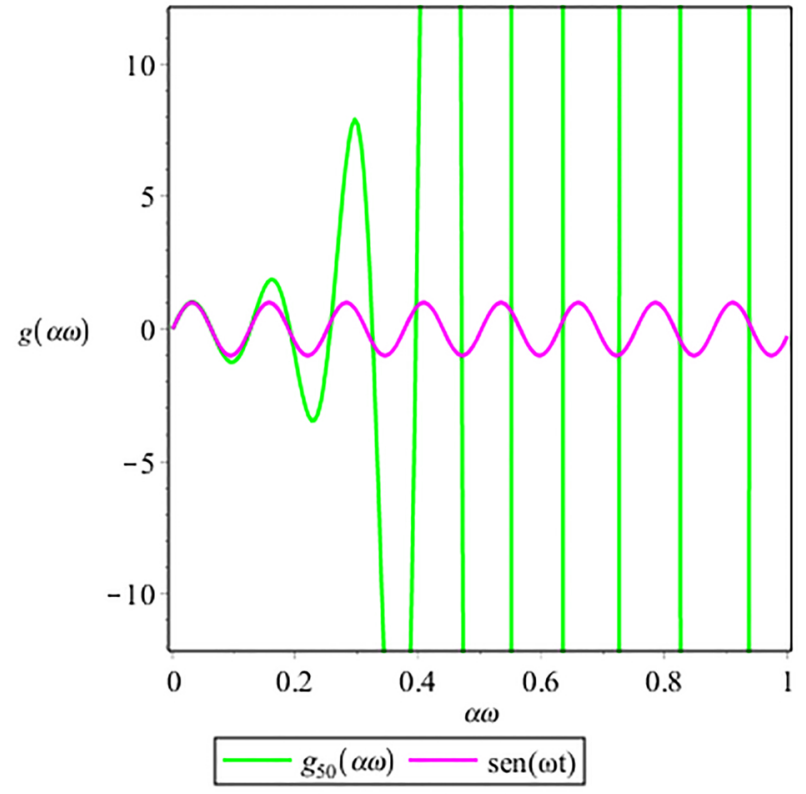

Figura 4: Amplitude $g(\alpha w)$ para $n=50$ e sua respectiva função trigonométrica. Assim como para $f(\alpha w)$, as amplitudes diminuem para $\alpha w$ maiores quando comparados com $n=25$.

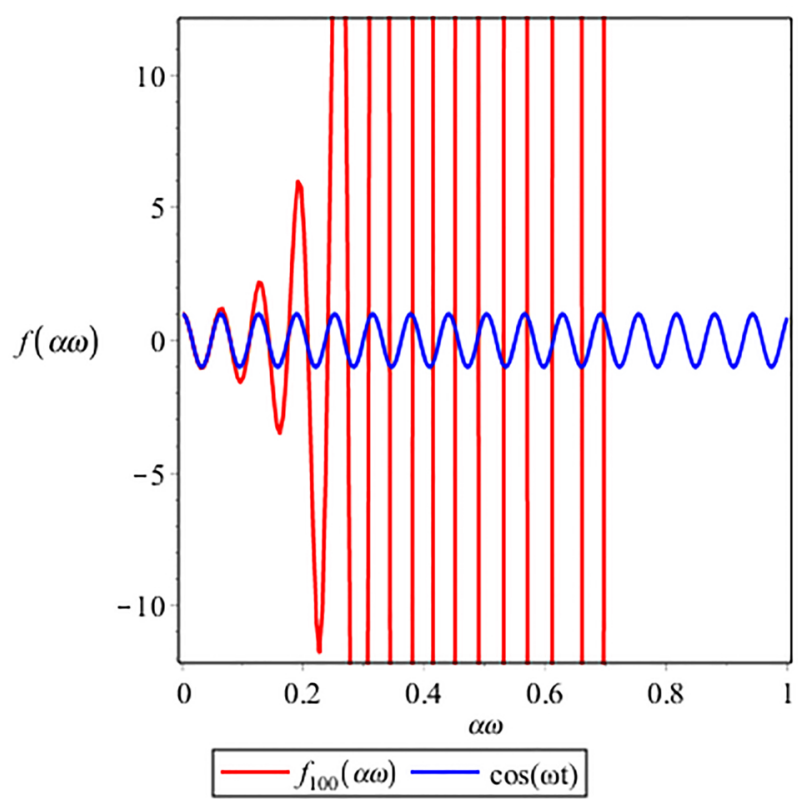

Figura 5: Amplitude $f(\alpha w)$ (que pode ser interpretada como $\frac{v_{100}}{v_{0}}$ quando $\left.x_{0}=0\right)$ e sua respectiva função trigonométrica.

as soluções 41 e 42 devem satisfazer duas condições para recuperarmos a solução do ohs $n>>1$ e $\alpha w n=$ $w\left(t_{n}-t_{0}\right)<<1$. Na mecânica um dos elementos constitutivos é assumir a existência de uma lei causal conectando os diversos estados do sistema 26]. Podemos então especificar $\alpha n$ pela reta real $(\alpha \in \mathbb{R}, n \in \mathbb{N} \Rightarrow \alpha n \in \mathbb{R})$ ou seja, é uma coordenada, em que cada valor de $\alpha n$ é chamado de instante de tempo. Consequentemente, as equações (41) e 42 podem ser interpretadas como solução do ohs. 


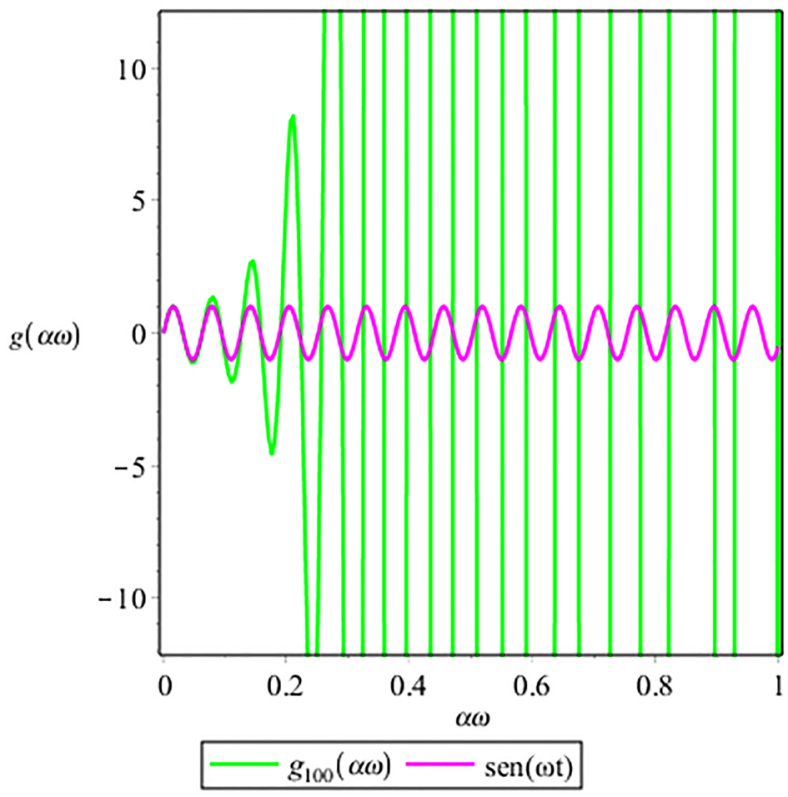

Figura 6: Amplitude $g(\alpha w)\left(\frac{x_{100} w}{v_{0}}\right.$ para $\left.x_{0}=0\right)$ e sua respectiva função trigonométrica.

Devemos destacar que a abordagem aqui proposta é de iteração e não de interação fundamental do ponto de vista físico.

\section{Considerações Finais}

Neste trabalho, foi proposto uma abordagem iterativa para a solução do ohs com o intuito de mostrar que é possível através de um método alternativo ao normalmente utilizado obter suas funções horárias. Não foi nosso intuito dar uma interpretação física ao método em si, o estamos considerando apenas como uma ferramenta matemática. Entretanto esta abordagem pode servir como base para analisar outros sistemas físicos, a saber as forças fundamentais. Não devemos esquecer,que o potencial harmônico, embora seja uma ferramenta extremamente útil em todos os ramos da física moderna, não é em si uma interação fundamental.

\section{Agradecimentos}

Ao Professor Manoelito Martins de Souza e ao Julio Cesar Azevedo da Silva pela colaboração, aos árbitros anônimos pelas valiosas sugestões e aos órgãos de fomento CNPq, CAPES e FAPES.

\section{Material suplementar}

O seguinte material suplementar está disponível online: Apêndice A

Apêndice B

\section{Referências}

[1] M.A. Moreira, N.T. Massoni e F. Ostermann, Rev. Bras. Ensino Fís. 29, 127 (2007).

[2] M.A. Moreira e I. Krey, Rev. Bras. Ensino Fís. 28, 353 (2006).

[3] A. Llancaqueo, M.C. Caballero e M.A. Moreira, Rev. Bras. Ensino Fís. 25, 399 (2003).

[4] V.O. Almeida e M.A. Moreira, Rev. Bras. Ensino Fís. 30, 1806 (2008).

[5] I.S. Araujo, E.A. Veit e M.A. Moreira, Rev. Bras. Ensino Fís. 26, 179 (2004).

[6] R.R. Oliveira e L. Ferracioli, Ens. Pesqui. Educ. Ciênc. 17, 685 (2015)

[7] M.E. de Andrade,Simulação e modelagem computacional com o software Modellus: aplicações práticas para o ensino de física (Editora Livraria da Física, São Paulo, 2016)

[8] C.H.S. Verbeno, R.M.A. da Silva, R. Maziero, T. Gomes e L. Ferracioli, Rev. Bras. Ensino Ciên. e Tecn. 924 (2016).

[9] G.S. Maciel,Proposta de uma Sequência Didática Sobre Tópicos de Física Quântica Através do Uso de Simulações Computacionais e da Determinação da Constante de Planck Com Leds Aplicado ao Ensino Médio. Dissertação de Mestrado, Universidade Federal do Espírito Santo, Espírito Santo, 2016.

[10] R.T. Prado e L. Ferracioli, Rev. Prof. Fís. 1, 1 (2017).

[11] J.C. dos Santos e A.G. Dickman, Rev. Bras. Ensino Fís. 41, 1 (2019).

[12] L.V. Soares, Aprendizagem Significativa Através da Construção de Experimentos Pelos Alunos do Ensino Médio Técnico.Dissertação de Mestrado, Universidade Federal do Espírito Santo, Espírito Santo, 2015.

[13] S. Bose, D. Home e S. Mal, Phys. Rev. Lett. 120, 210402 (2018).

[14] X. Xu, T. Purdy e J.M. Taylor, Phys. Rev. Lett. 118, 223602 (2017)

[15] N. Paul et al., Phys. Rev. Lett. 118, 032501 (2017).

[16] M.F. Bocko e R. Onofrio, Rev. Mod. Phys. 68, 755 (1996).

[17] G. Salerno, T. Ozawa, H.M. Price e I. Carusotto, Phys. Rev. B. 93, 085105 (2016).

[18] J.I. Jiménez-Aquino, R.M. Velasco e F.J. Uribe, Phys. Rev. E. 79, 061109 (2009).

[19] J.I. Jiménez-Aquino, R.M. Velasco e F.J. Uribe, Phys. Rev. E. 77, 051105 (2008).

[20] M. Teubner, Phys. Rev. A. 72, 042703 (2005).

[21] Y. Zhang, Rev. Bras. Ensino Fís. 37, 4314 (2015).

[22] H.M. Nussenzveig, Curso de Física Básica (Blucher, São Paulo, 2002), v. 1, p. 86.

[23] H.M. Nussenzveig, Curso de Física Básica (Blucher, São Paulo, 2002), v. 2, p. 40.

[24] B.R. Segatto, J.C.S. Azevedo e M.M. de Souza, J. Phys. A: Math. Gen. 365155 (2003).

[25] J. Stewart, Cálculo (Cengage Learning, São Paulo, 2014), v. 2 , p. 684 .

[26] A.E. de Santana, Rev. Bras. Ensino Fís. 41, e20180145 (2019). 